Casos Clínicos

Arch. Esp. Urol., 61, 5 (624-626), 2008

\section{URINOMA POR IATROGENIA URETERAL}

\author{
Javier Casasola Chamorro, Sonia Gutiérrez García' y \\ María Suárez Mayor?.
}

Servicio de Urología y Servicio de Ginecología'. Complejo Hospitalario de León. León. España.

Resumen.- OBJETIVOS: Presentar un caso de urinoma tras cirugía ginecológica y su resolución quirúrgica.

METODOS: Mujer de 46 años remitida a Urología por dolor en flanco tras histerectomía

RESULTADOS: EI TAC evidencia un urinoma, con el uréter incluido en dicha lesión

CONCLUSIONES: El tratamiento precoz con anastomosis termino terminal de los extremos del uréter lesionado solucionó el problema.

Palabras clave: Urinoma. Histerectomía. Lesión ureteral.
Summary.- OBJECTIVES: We present one case of urinoma after gynecologic surgery and the surgical repair.

METHODS: 46-year-old woman referred for urologic consultation for flank pain after hysterectomy.

RESULTS: CT scan showed the existence of an urinoma with the ureter included in the radiological lesion.

CONCLUSION: Early treatment after diagnosis with end-toend anastomosis solved the problem.

Keywords: Urinoma. Hysterectomy. Ureteral injury.

\title{
INTRODUCCIÓN
}

Las fístulas urinarias consideradas como comunicaciones patológicas de la vía urinaria y un órgano vecino son infrecuentes y un importante problema en la práctica quirúrgica sobre todo ginecológica y urológica $(1,2)$. El daño ureteral complica 10-30\% de las histerectomías radicales y entre $1,5-2,5 \%$ de los procedimientos ginecológicos (1) con la consiguiente necesidad de intervención del urólogo, siendo los urinomas colecciones de orina subperitoneales, secundarias a la sección inadvertida de un uréter o a la necrosis secundaria a una ligadura del mismo, con el consiguiente acúmulo de orina por debajo del peritoneo, que no drena a través de la herida o el fondo de saco vaginal (3).

\section{CASO CLÍNICO}

Mujer de 46 años de edad, con antecedente de endometriosis ovárica, a quien se practicó una histerectomía abdominal con doble anexectomía por endometriosis 45 días antes de acudir a consulta, remitida por su ginecólogo por presentar desde el postoperatorio inmediato dolor continuo en región iliaca y sacra derecha que mejoraba con antiinflamatorios y por objetivarse en una ecografía abdominal una discreta ectasia renal. A la exploración la paciente presenta dolor y defensa a la palpación profunda de fosa lumbar derecha y dolorimiento a la percusión y palpación de fosa lumbar izquierda, sugestivo de patología retroperitoneal.

Se realiza urografía intravenosa (UIV) y Tomografía computerizada (TAC) simultáneos, observándose riñón derecho funcionante con retraso en la captación y eliminación de contraste y borramiento de la línea de psoas derecho. En la TAC se objetiva imagen lobulada de contenido líquido y con aparente extravasación de contraste, estando el uréter incluido en dicha formación (Figuras 1 y 2). Con el diagnóstico de urinoma por ligadura o sección del uréter derecho y tras estudio preoperatorio se realiza, vía iliaca, ureterolisis y exéresis del urinoma, con anastomo- 


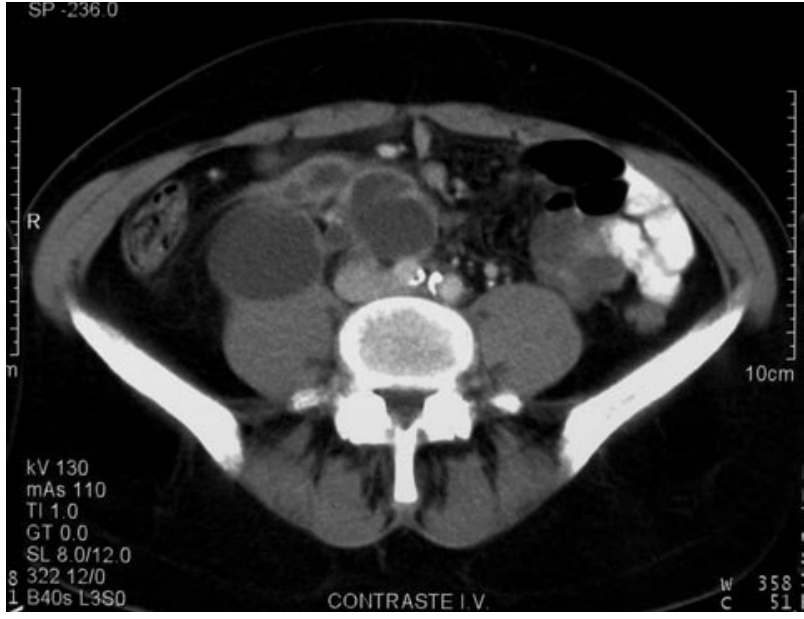

FIGURA 1. Visualización de imagen multilobulada compatible con urinoma.

sis termino-terminal del uréter tras refrescamiento de los bordes y liberación de ambos extremos. Se deja catéter doble J tutorizando el uréter que se retira al mes de la cirugía. La evolución de la paciente ha sido favorable sin complicaciones en el postoperatorio. En el control al año de la intervención se objetiva buena función renal con normalidad de la vía urinaria en UIV (Figura 3).

\section{DISCUSIÓN}

Las lesiones ureterales ocurren tras gran variedad de procedimientos ginecológicos, urológicos, vasculares o de cirugía general (4) siendo el $80 \%$ de las lesiones del uréter secundarias a cirugía y sólo un $20 \%$ secundarías a traumatismo externo (5). La mayoría no se detectan en un primer momento, presentándose posteriormente con dolor, fiebre, distensión abdominal, íleo, sepsis e incluso pérdida renal, siendo el retraso medio de detección de la lesión de 10 a 12 días $(4,6)$. El dolor en flanco y la fiebre, como ocurrió en nuestro caso, son los síntomas más frecuentes de lesión ureteral, siendo un 30\% de las lesiones asintomáticas. Sobre el momento de reparación de las lesiones fistulosas no existe consenso, siendo la experiencia y las preferencias del urólogo las que marcan el tratamiento. Sin embargo la tendencia actual defiende la reparación precoz, excepto en casos de fístulas complejas o secundarias a radioterapia (7).

Nuestro caso representa una magnífica oportunidad para valorar la intervención precoz ante un urinoma, frente a la postura de derivación y espera. El urinoma que presentaba la paciente no supuso gran dificultad quirúrgica y nos sirvió de referencia, como se aprecia en la TAC, para encontrar el uréter. Disecamos primero el urinoma en los planos más favorables, buscando mejorar el campo quirúrgico, y posteriormente tras incidir el urinoma y vaciarlo, resecando su pared fibrosa, llegar

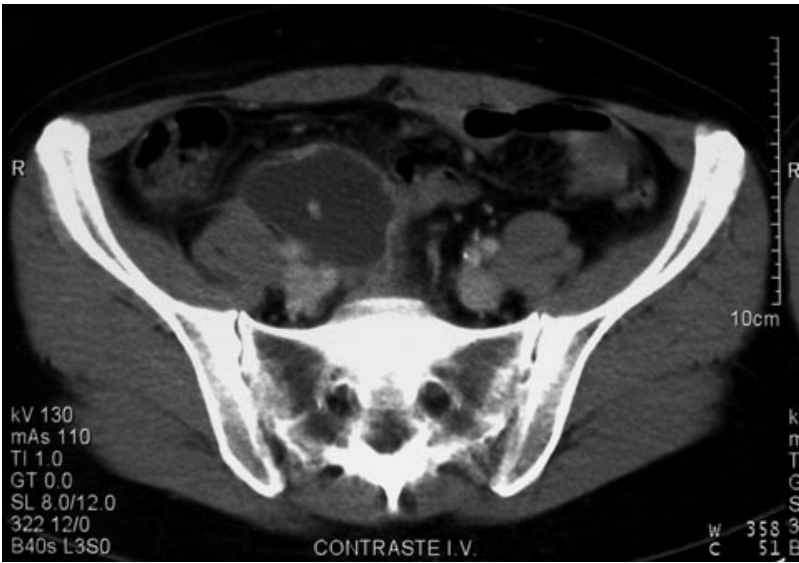

FIGURA 2. Urinoma con uréter en su parte central, remarcado por el contraste.

al uréter en el que posiblemente la lesión se debía a una ligadura en bloque con el pedículo ovárico. Resecamos ambos extremos hasta llegar a bordes cruentos y tras espatular ambos cabos realizamos la anastomosis, tutorizando el uréter y fijándolo a músculo psoas, fuera de la zona de urinoma residual. Creemos que en el tratamiento de las lesiones ureterales el primer paso debe ser

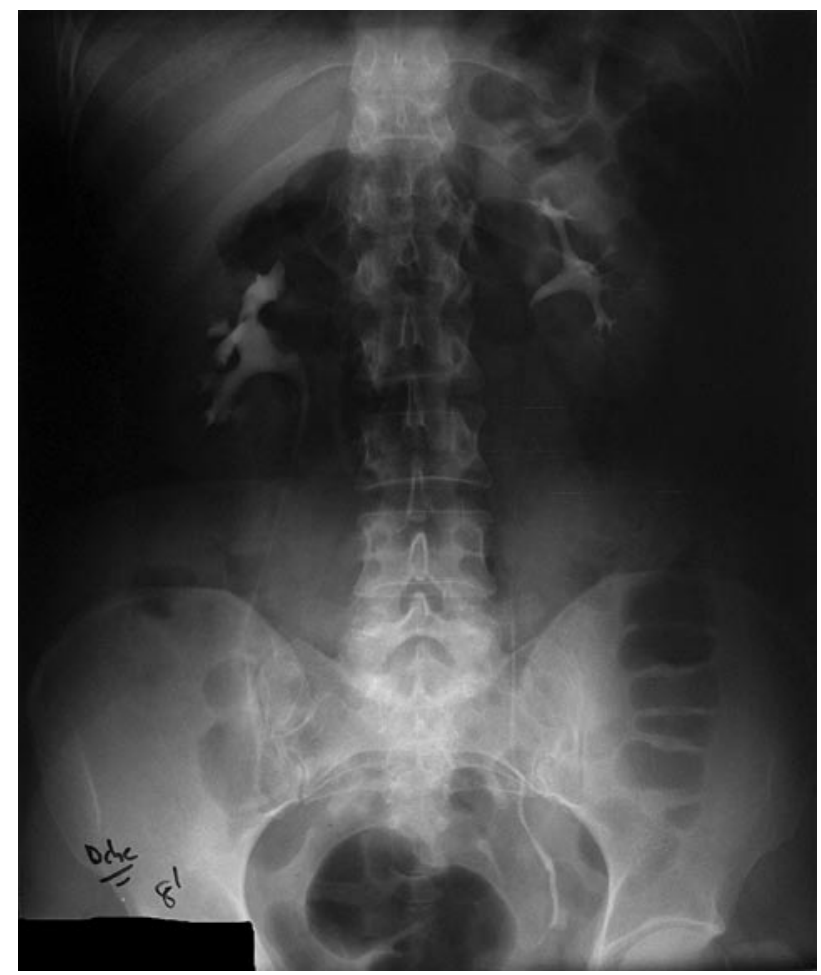

FIGURA 3. UIV. Normofunción postquirúrgica de vía urinaria. 
el intento de cateterización retrógrada en la fase más precoz posible tras la cirugía, cuando la proximidad - la afectación parcial del mismo permitan el paso de guías o catéteres. Si no es así, diferir durante meses la cirugía, utilizando nefrostomías, en pacientes con buen estado general, no aporta ningún beneficio, ya que los urinomas raramente están infectados y la derivación renal podría añadir iatrogenia, perdiéndose además longitud de uréter y teniendo peores condiciones en los extremos de anastomosis.

\section{CONCLUSIONES}

Animamos pues al tratamiento quirúrgico precoz de estas lesiones, disminuyendo el estrés al que están sometidos estos pacientes y los profesionales que acuden a nosotros tras la iatrogenia.

El tratamiento precoz con anastomosis termino-terminal de los extremos del uréter lesionado solucionó el problema.
Casos Clínicos

Arch. Esp. Urol., 61, 5 (626-630), 2008

\section{COEXISTENCIA DE DOS TUMORES GERMINALES SEMINOMATOSO Y NO SEMINOMATOSO CON UNA PRESENTACIÓN CLÍNICA INHABITUAL}

\author{
Pilar Soriano Sarrió, Isabel Chirivella y Samuel \\ Navarro Fos.
}

Departamento de Anatomía Patológica y Servicio de Hematooncología'. Hospital Clínico Universitario de Valencia. Valencia. España.

Resumen.- OBJETIVOS: La existencia de tumores mixtos
germinales no seminomatosos es un hecho frecuente en pa-
tología oncológica urológica. Sin embargo, la presencia
de componente seminomatoso y no seminomatoso en un
tumor germinal es una situación infrecuente.

MÉTODOS: Presentamos un caso de un seminoma puro testicular con metástasis ganglionar por carcinoma embrionario puro con un completo estudio inmunohistoquímico diferencial y evolución clínica del paciente.

RESULTADOS: Este caso corresponde a un varón de 34 años que acude a urgencias por notar una tumoración supraclavicular de $3 \mathrm{~cm}$. En TAC de control se detecta adenopatías múltiples de gran tamaño a nivel supracla-

Pilar Soriano Sarrió

Departamento de Anatomia Patológica

Hospital Clínico Universitario de Valencia

Avda. Blasco lbañez, 17

46010 Valencia. (España).

pilarsoriano 10@yahoo.es

Trabajo recibido: 12 de septiembre 2007. 